\title{
A Note on Class-Number One in Certain Real Quadratic and Pure Cubic Fields
}

\author{
By M. Tennenhouse and H. C. Williams*
}

\begin{abstract}
Let $p$ be any odd prime and let $h(p)$ be the class number of the real quadratic field $2(\sqrt{p})$. The results of a computer run to determine the density of the field $2(\sqrt{p})$ with $h(p)=1$ and $p<10^{8}$ are presented. Similar results are given for pure cubic fields $2(\sqrt[3]{p})$ with $p<10^{6}$.
\end{abstract}

1. Introduction. Let $p$ be any odd prime and let $h=h(p)$ be the class number of the quadratic field $2(\sqrt{p})$. It is well known that $h(p)$ is odd, but the problem of how frequently $h(p)=1$, although it goes back to Gauss, is still unsolved.

If we let $\pi(a, b ; x)$ denote the number of primes of the form $a+b k$ less than or equal to $x$ and $f(a, b ; x)$ denote the number of these primes $p$ for which $h(p)=1$, we find (see Lakein [5]) from a large table of Kuroda [4], that

$$
r(1,4 ; x)=f(1,4 ; x) / \pi(1,4 ; x)=.7765
$$

for $x=2776817$; that is, over $77 \%$ of all the primes $(\equiv 1(\bmod 4))$ up to 2776817 have $h(p)=1$. Indeed, according to the recent heuristic results of Cohen and Lenstra (see Cohen [1]), we would expect that $h(p)=1$ with probability 75446 .

In order to test this heuristic, we developed and ran a computer program which determined whether or not $h(p)=1$ for all primes $p<10^{8}$. In the next section of this note we give the results of this computer run. In the following section we present some data for certain pure cubic fields $2(\sqrt[3]{p})$ with $p<10^{6}$.

2. The Quadratic Case. In order to find $h(p)$, we made use of the well-known formula

$$
2 h R=\sqrt{\Delta} L(1, \chi),
$$

where $\Delta$ is the discriminant of $\mathscr{Q}(\sqrt{p}), R$ is the regulator, and $L(1, \chi)$ is the value of the Dirichlet $L$-function

$$
\sum_{n=1}^{\infty}\left(\frac{\Delta}{n}\right) \frac{1}{n^{s}}
$$

for $s=1$. To evaluate $L(1, \chi)$ we employed a routine similar to the SPEEDY routine mentioned in Shanks [8]. Most of the time needed to find $h(p)$ was taken up computing $R$. This was done by using the techniques developed by Lenstra [6] and Schoof [7] (see, also, Williams [10]). The implementation of these ideas permitted us

Received June 22, 1984

1980 Mathematics Subject Classification. Primary 12-04, 12A25, 12A30, 12 A50.

* Research supported by NSERC of Canada grant number A7649 and the I. W. Killam Programme.

1986 American Mathematical Society $0025-5718 / 86 \$ 1.00+\$ .25$ per page 
TABLE 1

\begin{tabular}{|c|c|c|c|c|c|c|}
\hline \multirow{2}{*}{$x$} & \multicolumn{3}{|c|}{$a=-1, b=4$} & \multicolumn{3}{c|}{$a=1, b=4$} \\
\cline { 2 - 7 } & $\pi(a, b ; x)$ & $f(a, b ; x)$ & $r(a, b ; x)$ & $\pi(a, b ; x)$ & $f(a, b ; x)$ & $r(a, b ; x)$ \\
\hline 5000000 & 174319 & 134661 & .7724975 & 174193 & 134862 & .7742102 \\
\hline 10000000 & 332398 & 255697 & .7692495 & 332180 & 256345 & .7717051 \\
\hline 15000000 & 485429 & 372854 & .7680917 & 485274 & 373925 & .7705441 \\
\hline 20000000 & 635436 & 487699 & .7675029 & 635170 & 488752 & .7694821 \\
\hline 25000000 & 783173 & 600560 & .7668293 & 783059 & 602016 & .7688003 \\
\hline 30000000 & 929079 & 712172 & .7665355 & 928779 & 713887 & .7686296 \\
\hline 35000000 & 1073601 & 822569 & .7661775 & 1073173 & 824136 & .7679433 \\
\hline 40000000 & 1216966 & 932017 & .7658529 & 1216687 & 933970 & .7676337 \\
\hline 45000000 & 1359235 & 1040345 & .7653900 & 1358924 & 1042888 & .7674366 \\
\hline 50000000 & 1500681 & 1148210 & .7651259 & 1500452 & 1151039 & .7671282 \\
\hline 55000000 & 1641343 & 1255778 & .7650917 & 1640856 & 1258288 & .7668485 \\
\hline 60000000 & 1781444 & 1362483 & .7648194 & 1780670 & 1365129 & .7666378 \\
\hline 65000000 & 1920648 & 1468646 & .7646617 & 1919905 & 1471506 & .7664472 \\
\hline 70000000 & 2059345 & 1574494 & .7645605 & 2058718 & 1577494 & .7662506 \\
\hline 75000000 & 2197469 & 1679748 & .7644012 & 2196834 & 1682861 & .7660392 \\
\hline 80000000 & 2335008 & 1784833 & .7643798 & 2334373 & 1787874 & .7658904 \\
\hline 85000000 & 2472052 & 1889752 & .7644467 & 2471678 & 1892924 & .7658457 \\
\hline 90000000 & 2608560 & 1993991 & .7644029 & 2608393 & 1997296 & .7657189 \\
\hline 95000000 & 2745067 & 2098012 & .7642844 & 2744681 & 2101465 & .7656500 \\
\hline 100000000 & 2880950 & 2201430 & .7641333 & 2880504 & 2205112 & .7655299 \\
\hline
\end{tabular}

to evaluate $R$ much more rapidly than was done in Williams and Broere [11]. Indeed, without this innovation we would not have been able to complete our calculations because of time constraints. If we define

$$
r(a, b ; x)=f(a, b ; x) / \pi(a, b ; x),
$$

the results of running our program are summarized in Table 1.

Notice that the value of $r(a, b ; x)$ in both cases is tending to decrease more slowly as $x$ increases. These results are certainly consistent with the heuristic we get from [1].

3. The Pure Cubic Case. Let $H(p)$ denote the class number of $\mathscr{2}(\sqrt[3]{p})$. In order for $H(p)=1$, we must have $p=3$ or $p \equiv-1(\bmod 3)$ (Honda [3]); also, it has been noted by Eisenbeis, Frey, and Ommerborn [2] that $H(p)$ tends to be 1 more frequently for $p \equiv-1(\bmod 9)$, an observation that was tested empirically by Williams and Shanks [13]. Thus, in the cubic case we performed our computations on the primes in each of the residue classes $-1,2,5(\bmod 9)$.

Let $F(a, b ; x)$ be the number of primes $p$ of the form $a+b k$ less than or equal to $x$ for which $H(p)=1$, and put $R(a, b ; x)=F(a, b ; x) / \pi(a, b ; x)$. The results of our computer runs for the pure cubic case are given in Tables 2 and 3 . These tables were computed by making use of the algorithms given in Williams, Dueck and 
TABLE 2

\begin{tabular}{|c|c|c|c|}
\hline \multirow{2}{*}{$x$} & \multicolumn{3}{|c|}{$a=-1, b=9$} \\
\cline { 2 - 4 } & $\pi(a, b ; x)$ & $F(a, b ; x)$ & $R(a, b ; x)$ \\
\hline 200000 & 2993 & 1827 & .6104 \\
\hline 250000 & 3671 & 2240 & .6102 \\
\hline 300000 & 4337 & 2627 & .6057 \\
\hline 350000 & 4992 & 3041 & .6092 \\
\hline 400000 & 5650 & 3437 & .6083 \\
\hline 450000 & 6287 & 3820 & .6076 \\
\hline 500000 & 6924 & 4199 & .6064 \\
\hline 550000 & 7550 & 4568 & .6050 \\
\hline 600000 & 8174 & 4940 & .6044 \\
\hline 650000 & 8802 & 5332 & .6058 \\
\hline 700000 & 9416 & 5701 & .6055 \\
\hline 750000 & 10033 & 6065 & .6045 \\
\hline 800000 & 10670 & 6435 & .6031 \\
\hline 850000 & 11282 & 6789 & .6018 \\
\hline 900000 & 11890 & 7157 & .6019 \\
\hline 950000 & 12487 & 7523 & .6025 \\
\hline 1000000 & 13094 & 7903 & .6036 \\
\hline
\end{tabular}

TABLE 3

\begin{tabular}{|c|c|c|c|c|c|c|}
\hline \multirow{2}{*}{$x$} & \multicolumn{3}{|c|}{$a=2, b=9$} & \multicolumn{3}{c|}{$a=5, b=9$} \\
\cline { 2 - 7 } & $\pi(a, b ; x)$ & $F(a, b ; x)$ & $R(a, b ; x)$ & $\pi(a, b ; x)$ & $F(a, b ; x)$ & $R(a, b ; x)$ \\
\hline 200000 & 2994 & 1200 & .4008 & 2988 & 1293 & .4327 \\
\hline 250000 & 3679 & 1440 & .3914 & 3677 & 1559 & .4240 \\
\hline 300000 & 4328 & 1699 & .3926 & 4341 & 1845 & .4250 \\
\hline 350000 & 5007 & 1965 & .3925 & 4999 & 2096 & .4193 \\
\hline 400000 & 5651 & 2224 & .3936 & 5647 & 2366 & .4190 \\
\hline 450000 & 6281 & 2471 & .3934 & 6296 & 2628 & .4174 \\
\hline 500000 & 6916 & 2755 & .3984 & 6945 & 2873 & .4137 \\
\hline 550000 & 7541 & 2987 & .3961 & 7577 & 3124 & .4123 \\
\hline 600000 & 8176 & 3223 & .3942 & 8204 & 3366 & .4103 \\
\hline 650000 & 8829 & 3472 & .3932 & 8806 & 3607 & .4096 \\
\hline
\end{tabular}

Schmid [12]. Since precision problems in our implementation of these algorithms occur later for Dedekind type 2 fields than for type 1 fields, we were able to compute Table 2 out somewhat further than Table 3.

In Table 2 we notice that the surprisingly flat behavior of $R(-1,9 ; x)$ for $1.5 \times 10^{5}<x<2 \times 10^{5}$, which was pointed out in [13], persists (although it does tend to decrease slightly) up to $10^{6}$. These results, then, are still consistent with the 
conjectures made in [13]. In Table 3 we see that the values of $R(2,9 ; x)$ and $R(5,9 ; x)$ are coming closer together. This indicates that the peculiar behavior of these ratios for $x<2 \times 10^{5}$ noted in Williams [9] was simply a result of this range of $x$ values being too small.

Department of Computer Science

University of Manitoba

Winnipeg, Manitoba

Canada R3T 2N2

1. H. Cohen, "Sur la distribution asymptotique des groupes de classes," C. R. Acad. Sci. Paris Sér. I Math., v. 296, 1983, pp. 245-246.

2. H. Eisenbeis, G. Frey \& B. Ommerborn, "Computation of the 2-rank of pure cubic fields," Math. Comp., v. 32, 1978, pp. 559-569.

3. T. Honda, "Pure cubic fields whose class numbers are multiples of three," J. Number Theory, v. 3. 1971, pp. 7-12.

4. S. Kuroda, "Table of class numbers $h(p)>1$ for quadratic fields $Q(\sqrt{p}), p \leqslant 2776817$," Math. Comp., v. 29, 1975, pp. 335-336, UMT File.

5. R. B. LaKein, "Computation of the ideal class group of certain complex quartic fields, II," Math. Comp., v. 29, 1975, pp. 137-144.

6. H. W. Lenstra, JR., On the Calculation of Regulators and Class Numbers of Quadratic Fields, London Math. Soc. Lecture Note Series, no. 56, 1982, pp. 123-150.

7. R. SchоOF, "Quadratic fields and factorization," Computational Methods in Number Theory, Part II, Math. Centrum Tracts, No. 155, Amsterdam, 1983, pp. 235-286.

8. D. Shanks, Class Number, A Theory of Factorization, and Genera, Proc. Sympos. Pure Math., Vol. 20 (1969 Institute on Number Theory), Amer. Math. Soc., Providence, R. I., 1971, pp. 415-440.

9. H. C. Williams, "Improving the speed of calculating the regulator of certain pure cubic fields," Math. Comp., v. 35, 1980, pp. 1423-1434.

10. H. C. Williams. "Continued fractions and number-theoretic computations" (Proc. Number Theory

Conf. Edmonton 1983), Rocky Mountain J. Math., v. 15, 1985, pp. 621-655.

11. H. C. Williams \& J. Broere, "A computational technique for evaluating $L(1, \chi)$ and the class number of a real quadratic field," Math. Comp., v. 30, 1976, pp. 887-893.

12. H. C. Williams, G. W. Dueck \& B. K. Schmid, "A rapid method of evaluating the regulator and class number of a pure cubic field," Math. Comp., v. 41, 1983, pp. 235-286.

13. H. C. Williams \& D. Shanks, "A note on class-number one in pure cubic fields," Math. Comp., v. 33, 1979. pp. 1317-1320. 